\title{
Evaluating the Impacts of Real-Time Pricing on the Usage of Wind Generation*
}

\author{
Ramteen Sioshansi and Walter Short
}

March 15, 2008

\begin{abstract}
One of the impediments to large-scale use of wind generation within power systems is the non-dispatchability and its variable and uncertain real-time availability. Operating constraints on conventional generators such as minimum generation points, forbidden zones, and ramping limits as well as system constraints such as power flow limits and ancillary service requirements may force a system operator to curtail wind generation in order to ensure feasibility. Furthermore, the pattern of wind availability and electricity demand may not allow wind generation to be fully utilized in all hours. One solution to these issues, which could reduce these inflexibilities, is the use of real-time pricing (RTP) tariffs which can both smooth-out the diurnal load pattern in order to reduce the impact of binding unit operating and system constraints on wind utilization, and allow demand to increase in response to the availability of costless wind generation. We use and analyze a detailed unit commitment model of the Texas power system with different estimates of demand elasticities to demonstrate the potential increases in wind generation from implementing RTP.
\end{abstract}

\section{Introduction}

One of the difficulties presented by the use of wind and some other renewable sources of electricity is the fact that their actual available real-time generation can be variable and uncertain ex ante, limiting their dispatchability and dependability. For example, the availability of photovoltaic- or concentrating-solar energy is governed by the ambient sunlight, which is outside human control. Moreover, in some regions the availability of such a variable renewable energy resource can be negatively correlated with electricity demand.

Because supply and demand of electricity must be perfectly balanced at all times and most storage technologies are costly, the pattern of variable renewable energy supply and electric loads may sometimes preclude these energy sources from serving demand and at other time require their generation to be curtailed when output exceeds net load. These issues are further confounded by constraints on the operations of conventional and other dispatchable generation units (e.g. minimum operating points, forbidden generating zones, minimum up and down times, and ramp constraints) and on the power system (e.g. transmission constraints and ancillary service (AS) requirements). Denholm and Margolis (2007) gives the example of the Danish system, which has a large installed base of wind generators. The Danish system relies on combined heat and power thermal generators for heating, and as such many

${ }^{*}$ The authors would like to thank seminar participants at the National Renewable Energy Laboratory, Armin Sorooshian, David Hurlbut, Brendan Kirby, and Shmuel Oren for helpful suggestions and discussions. Tony Grasso, Paul Denholm, Ray George, Isabel Flores, and Dana Showalter provided invaluable assistance in gathering ERCOT market and wind generation data. 
thermal plants must be constantly kept online to serve heating needs. As a result, wind generation must often be curtailed on cold windy nights when wind generation could potentially be quite high but heating loads require keeping thermal generators online. Even in systems which do not rely on combined heat and power, the limited flexibility of the power system may require curtailment of renewable generation. Overnight they can be displaced by baseload units, which are normally kept online due to slow and expensive startups and are often kept close to their minimum operating points. During the day they can be displaced by mid-merit units that are starting- and ramping-up in the morning shoulder and ramping-down in the evening shoulder, due to midday peaks. Transmission constraints can substantially limit renewable energy production, especially in systems in which wind or solar resources are sited away from load pockets. In addition to these system inflexibility issues, day- and hour-ahead uncertainty in the realtime availability of variable renewable energy resources may require increasing AS procurements from dispatchable generators in order to ensure system reliability, potentially increasing the displacement of renewable resources due to operating constraints on the dispatchable generators used.

In many power systems today, these issues are largely muted by the fact that variable renewable energy resources account for a relatively small portion of total energy supplied. In the California ISO's control area, for instance, wind generation rarely exceeded 1,100MW in 2006 which is approximately $4 \%$ of the average system load of $27,406 \mathrm{MW}$. When wind and other variable energy resources account for such a small fraction of generation, their non-anticipativity will tend to have minimal effects on system reliability. With renewable portfolio standards coming into force and other market pressures increasing investments and reliance on these energy resources, these system operations issues will slowly become more prevalent. One potential way of overcoming these issues would be to alter the load pattern in such a way that electricity demand more closely follows supply of variable energy resources by leveraging real-time demand response. For example, flattening the daily peak may reduce the displacement of renewables by rampconstrained units, shifting day-time loads to hours later in the night may increase the system's flexibility to take renewable generation overnight, and using locational price differences to increase loads in areas with an abundant supply of renewable generation and reduce it elsewhere can increase wind utilization in a transmission-constrained system.

Over the last few years a number of authors have advocated real-time pricing (RTP) of electricity, in which retail electric prices change frequently to reflect changes in the supply of electricity and the cost of serving load (they typically suggest rates change hourly or sub-hourly since the cost of service can vary significantly on this timescale). These authors have typically advocated RTP for standard economic reasons, such as increasing social welfare by having consumers face the actual marginal cost of electricity service, or decreasing generators' market power by making demand more elastic. Borenstein (2002) suggests that the demand response resulting from RTP could have lessened the severity of the 2000-2001 California electricity crisis, while Borenstein (2005) and (2005) simulate the efficiency gains from RTP. These and other simulations generally show that RTP has the effect of changing the diurnal load pattern by flattening peaks and shifting those loads to off-peak hours, since peak prices tend to be higher than the fixed retail rates customers would otherwise face whereas off-peak prices are lower - which is the exact change in the load pattern which may increase system flexibility and allow greater use of renewable energy resources. The use of locational prices can help alleviate transmission bottlenecks and further 're-shape' the load pattern in different parts of the transmission network to more-closely follow the availability of renewable energy.

In this paper we use a detailed unit commitment model with historical system, market, and wind availability data from 2005 in the Electricity Reliability Council of Texas (ERCOT) system, to simulate the potential for RTP to increase the utilization of large-scale wind farms. We demonstrate that introducing demand response increases both the percentage of total load which is served by wind generation, and the percentage of potential wind generation 
which is actually used in real-time. We further demonstrate that putting commercial and industrial C\&I customers only on RTP tariffs while keeping residential customers on fixed rates can achieve most of the gains from RTP, which may be a more feasible alternative, given the costs of installing interval meters on residential customers. The remainder of this paper proceeds as follows: Section 2 describes the models, data, and assumptions underlying our analysis, section 3 summarizes the results of our simulations, and section 4 concludes.

\section{Model and Data}

Our analysis is based on a competitive unit commitment model of the ERCOT system, the details of which are given in the appendix 1 Our simulations were conducted using a set of 165 days from January to October of 2005, for which all the requisite datasets were available. Each day in the sample was simulated using two unit commitment models, one of which simulated a day-ahead commitment and the other a real-time dispatch 2

The day-ahead unit commitment was solved using an hourly time-step for the commitment and dispatch variables and day-ahead forecasts of hourly loads, transmission capacities, and availability of wind generation. In addition to load-balance constraints the model included load-based AS requirements. Our model only accounted for spinning and non-spinning reserves (i.e. regulation services were not modeled), and assumed a 15-minute response time for both AS products to determine generators' capabilities based on their hourly ramp rates. We assumed a total reserve requirement of $9 \%$, at least half of which must be met by spinning reserves. Wind generators were assumed not to be qualified to provide AS, and we further assumed the system operator (SO) may 'derate' forecasted wind generation schedules in determining AS requirements, due to uncertainty in their real-time availability. More precisely, we formulated the AS requirement in each hour, $t$, as 3

$$
\rho w_{t}+g_{t}+a_{t} \geq(1+r) l_{t},
$$

where $\rho \in[0,1]$ is the day-ahead wind schedule rating factor, $r \geq 0$ is the fraction of load which must be procured in $\mathrm{AS}, w_{t}, g_{t}, a_{t}$ are the total day-ahead wind generation, conventional generation, and AS schedules, respectively, and $l_{t}$ is the load. The wind schedule rating factor can be thought of analogously as increasing day-ahead AS requirements in proportion to day-ahead wind schedules. To see this, note that the hourly load-balance constraint is given by 4

$$
w_{t}+g_{t}=l_{t}
$$

Combining these two equations, the AS requirement becomes:

$$
a_{t} \geq r l_{t}+(1-\rho) w_{t}
$$

the right-hand side of which is decreasing in $\rho$. We simulated a set of rating factors ranging from 0.1 to 1.

\footnotetext{
${ }^{1}$ See Muckstadt and Koenig (1977) and Hobbs, Rothkopf, O'Neill, and Chao (2001) for a background on unit commitment models and solution techniques.

${ }^{2}$ In addition to these we also used an 'initialization' unit commitment, which had a three-day planning horizon (with the day being studied in the middle), to fix the starting and ending commitment and initial minimum up and down time constraints on the generating units. Importantly, the inclusion of the third day ensures baseload units are not taken offline in the evening of the day being studied, as might happen with a one-day planning horizon since the model would not see the need to keep these unit online to serve the following day's load.

${ }^{3}$ As mentioned above, there are actually two sets of constraints of this form for both spinning and non-spinning reserves.

${ }^{4}$ Because a zonal power flow model is included in our formulation (which is described in further detail later), there is in fact a loadbalance constraint for each transmission zone, which also ensures network flows do not violate the transfer capacity of any transmission line. The constraint given here is actually the sum of the individual zonal load-balance constraints.
} 
Once the day-ahead commitments of the units were determined, these were then fixed (except for units assigned non-spinning reserves, which could be started up) and a dispatch model was solved with 15-minute time-steps and actual load, transmission capacity, and wind generation availability data, representing real-time system operations. The dispatch model did not place upper and lower bounds on generators' outputs based on the day-ahead schedule, rather the $\mathrm{SO}$ is assumed to choose a feasible social welfare-maximizing dispatch (given the fixed commitments). As such, the day-ahead market is assumed to only make binding commitments as opposed to dispatches. Both the day-ahead unit commitment and real-time dispatch models were formulated as mixed-integer programs using GAMS and solved using cplex 9.0.

Generator costs were modeled as consisting of three parts - a startup cost, which is incurred whenever a generator is started up; a spinning no-load cost, which is incurred whenever a generator is online; and a non-decreasing stepped variable generating cost function. Generator capacities, minimum generating points, ramp rates, AS capabilities, minimum up and down times, and must-run requirements were included in the model formulation as well. Generator costs were computed using heat rate values, fuel and emission permit prices, and variable operation and maintenance costs obtained from Global Energy Decisions and Platts Energy. Generator constraint parameters were also obtained from the same sources.

Power flows within the network were represented using the linearized zonal DC power-flow model used in ERCOT's congestion management system - consisting of five zones and six commercially significant constraints (CSCs). Power transfer distribution factors (PTDFs) between zonal injections and CSCs, and total transfer capacities (TTCs) on each CSC were obtained from ERCOT. The PTDF data consisted of monthly averages, whereas TTCs were the monitored limit on each CSC, reported at 15-minute intervals.

Simulations without RTP were conducted using actual historical load data, which were obtained from the Public Utility Commission of Texas (PUCT). The PUCT data included both day-ahead load forecasts as well as actual real-time loads reported at 15-minute intervals. Simulations with RTP were conducted by constructing a priceelastic demand function, and formulating the unit commitment objective to maximize social surplus as opposed to minimizing cost. Following Borenstein and Holland (2005) we assume that cross-price elasticities between demands in different periods are zero 5 As done in Boresnstein, Bushnell, and Knittel (1997), we construct the demand function by assuming a fixed elasticity and calibrating the demand function so it goes through the locus defined by the actual historical load and the retail price of electricity - since the actual historical loads reveal demand for electricity at the historical retail price. Because different customer types face different retail prices, loads were broken down into industrial, commercial, and residential segments, based on the proportion of total electricity demand in Texas in 2005 of the three sectors, as reported by the Energy Information Administration (EIA). The EIA also provided average retail rates for Texas in 2005 for the three customer segments, which we used in determining the retail price of electricity. Because these rates included non-energy costs such as distribution, metering, and nuclear decommissioning, we subtracted these components from the EIA-reported rates, based on PUCT tariffs. We conducted simulations with a set of demand functions representing elasticities ranging from -0.10 to -0.30 . Each demand function was approximated as a 100-piece step function.

Wind generators are assumed to operate at no cost. Their generation is limited by both the installed nameplate capacity at each wind site, as well as prevailing wind speeds. In order to model large-scale wind investments in our simulations, we include all wind generators which are currently built, under construction, or proposed to be built by 2011 - totaling $9,864 \mathrm{MW}$ of nameplate capacity (which represents approximately $10 \%$ of the system nameplate capacity of $91,009 \mathrm{MW}$ ). Wind generators were modeled as belonging to one of the twenty competitive renewable

\footnotetext{
${ }^{5}$ We discuss the implications of this assumption further in section 4
} 
energy zones (CREZ) within ERCOT6 6 based on their geographic location. The CREZ are regions within Texas that have been identified as prime wind generation sites, into which the state is encouraging investment by increasing transmission capacity. Wind availability data is based on a meso-scale model provided by AWS Truewind, which specifies hourly potential wind generation within different sites in each CREZ. Day-ahead forecasts of and real-time wind availability are generated by randomly sampling empirical distribution functions fitted to the meso-scale model data. Figure 1 shows the transmission zones and CREZ within the ERCOT system.

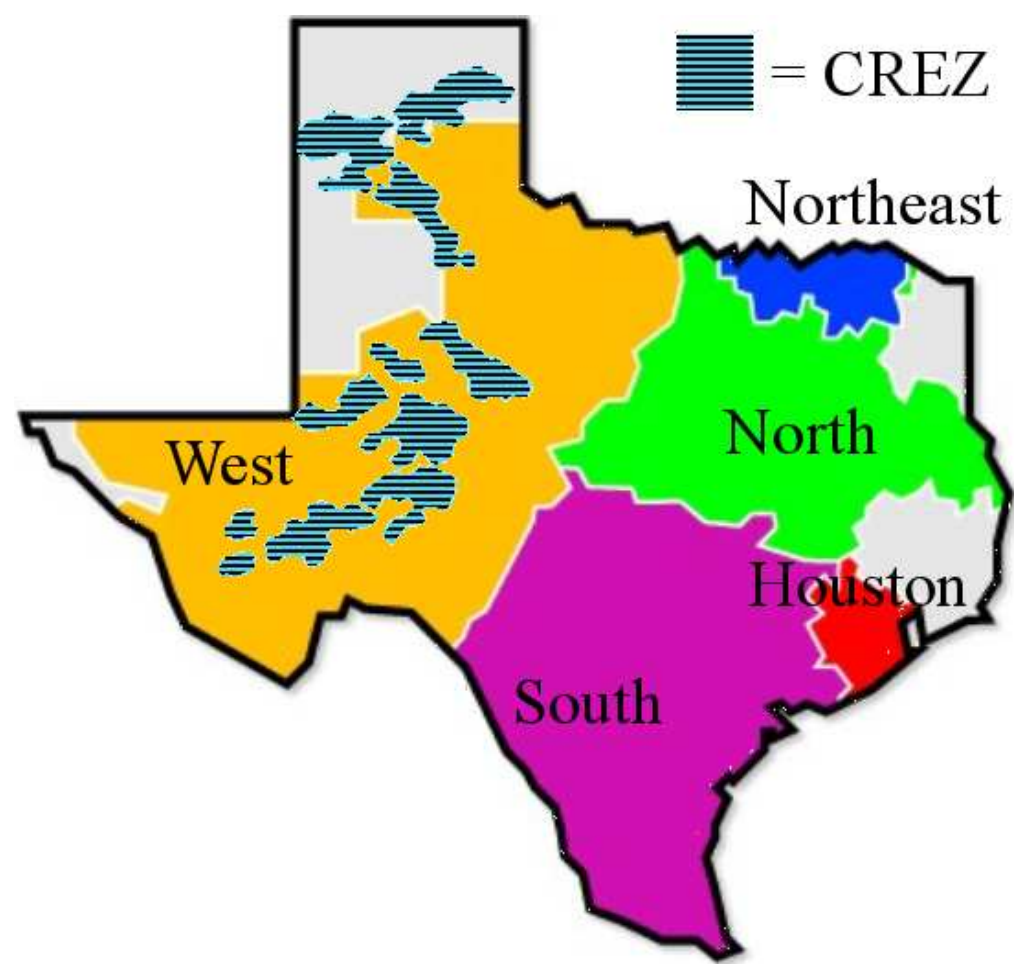

Figure 1: Map of ERCOT Congestion Zones and CREZ.

\section{Simulation Results}

Tables 1 and 2 summarize the results of our simulations, showing wind utilization averages for the days simulated. Table 1]shows the average percentage of potential wind generation that is actually dispatched in real-time for different demand elasticity and day-ahead wind schedule ratings, whereas table 2 reports the average percentage of total load that is served by wind generation. Our results show substantive increases of up to $7 \%$ in the usage of potential wind generation, which translates into an increase of up to $1 \%$ in the fraction of load served by wind, or approximately a $10 \%$ increase in wind generation's contribution to load. The results also show very little dependence on the treatment of wind forecasts in determining AS requirements - with less than a $0.4 \%$ difference between $10 \%$ and $100 \%$ ratings of day-ahead wind schedules.

The wind utilization rates in table 1 are lower than those typically reported in other wind resource studies due to one or more CSCs limiting exports from the West zone, which is where most of the wind generation is sited, into

\footnotetext{
${ }^{6}$ There are a total of twenty-five CREZ in Texas, but only twenty of them are within the ERCOT control area. We assume wind generators sited within those other five CREZ are not interconnected with the ERCOT system.
} 
Table 1: Average Percentage of Potential Wind Generation Utilized Wind Schedule Rating

\begin{tabular}{ll|lll} 
& & 0.1 & 0.5 & 1.0 \\
\cline { 2 - 5 } Elasticity & -0.00 & 75.00 & 75.23 & 75.24 \\
& -0.10 & 77.53 & 77.79 & 77.80 \\
& -0.20 & 79.86 & 80.10 & 80.11 \\
& -0.30 & 82.01 & 82.22 & 82.24
\end{tabular}

Table 2: Average Percentage of Load Served by Wind Generation Wind Schedule Rating

\begin{tabular}{ll|lll} 
& & 0.1 & 0.5 & 1.0 \\
\cline { 2 - 5 } Elasticity & -0.00 & 8.89 & 8.91 & 8.91 \\
& -0.10 & 9.20 & 9.25 & 9.28 \\
& -0.20 & 9.47 & 9.53 & 9.57 \\
& -0.30 & 9.71 & 9.77 & 9.83
\end{tabular}

load pockets in eastern Texas 7 Indeed, when transmission constraints are relaxed wind utilization rates rise to above 90\%. As tables 3 and 4 show, one effect of introducing demand response is to decrease the impact of these CSCs by using locational price differences to decrease demand in the eastern zones and increase it in the West zone when transmission constraints would otherwise be binding - thereby reducing the number of periods in which exports are constrained and correspondingly increasing wind utilization 8

Table 3: Number of 15-Minute Intervals in Which Exports From the West Zone Are Binding Wind Schedule Rating

\begin{tabular}{ll|lll} 
& & 0.1 & 0.5 & 1.0 \\
\cline { 2 - 5 } Elasticity & -0.00 & 10432 & 10320 & 10323 \\
& -0.10 & 10364 & 9865 & 9698 \\
& -0.20 & 10361 & 9848 & 9775 \\
& -0.30 & 10208 & 9869 & 9878
\end{tabular}

Figure 2 compares potential and actual wind generation patterns with and without RTP on 14 April, in which West zone exports become non-binding in several periods with RTP. The RTP run assumed a demand elasticity of -0.30 , and both runs assumed day-ahead wind schedules are fully rated. The figure highlights the fact that RTP increases wind utilization both by relieving congestion, and also increases usage in periods in which exports remain constrained.

RTP increases the scheduled dispatch of wind generators in transmission-constrained periods by smoothing out the diurnal load pattern and decreasing the extent to which operating constraints on conventional generators are binding. Figure 3 plots the load pattern with and without RTP and the corresponding increase in wind generation

\footnotetext{
${ }^{7}$ It bears noting that the values reported in table 1 may actually overstate wind utilization rates because our zonal model does not represent a number of constraints within the West zone, for instance the Crane substation, which tend to be more binding on wind exports than the six CSCs represented in ERCOT's zonal load-flow model.

${ }^{8}$ One reason we study a system with wind generation capacities expanded to 2011 levels but use the 2005 transmission network is to better capture the effects of RTP on increasing wind usage when transmission constraints would otherwise be binding.
} 
Table 4: Total Increase in Wind Generation (in MW) During 15-Minute Intervals in Which Exports From the West Zone Becomes Non-binding Under RTP

\begin{tabular}{ll|lll} 
& \multicolumn{4}{c}{ Wind Schedule Rating } \\
& & 0.1 & 0.5 & 1.0 \\
\cline { 2 - 5 } Elasticity & -0.10 & 1003 & 7154 & 8697 \\
& -0.20 & 1408 & 9256 & 11686 \\
& -0.30 & 804 & 11528 & 13356
\end{tabular}

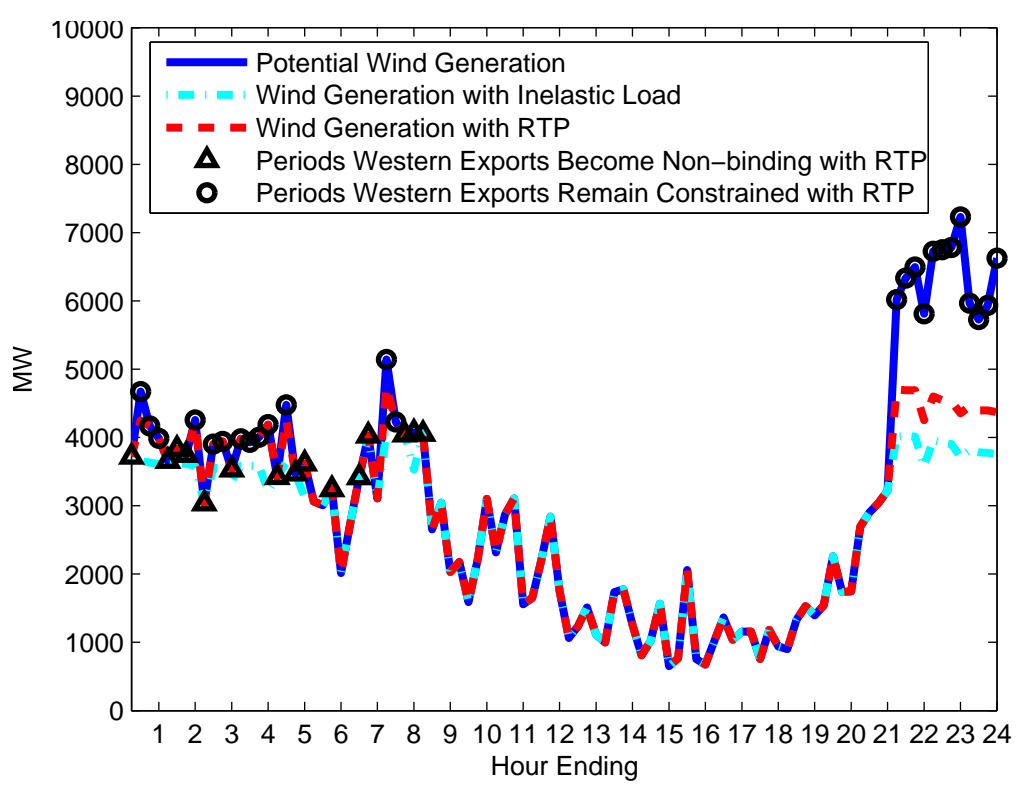

Figure 2: Wind Generation Patterns With and Without Real-Time Pricing.

(given as a percentage of total wind generation available) from our simulation of system operations on 20 October, assuming a demand elasticity of -0.30 and that wind schedules are fully rated day-ahead 9 The figure highlights the extent to which RTP smooths out the load pattern, by both substantially decreasing the peak and plateauing the midday loads, and slightly increasing early morning demand. The net effect of this on system operations is that the entire load can be served by 214 conventional generators, as opposed to the 219 that must be committed without demand response. Moreover, the conventional generators are significantly less ramp-constrained, which allows more wind generation to offset conventional generation. Tables 5 and 6 compare the number of units that are rampconstrained in real-time for the same two sets of runs from 20 October (hours without binding ramp constraints are excluded). The ramp down constraints, which all occur in the early morning and late evening hours, are caused by generators having to decrease output due to decreasing off-peak loads, and cause wind curtailments because conventional generators cannot reduce their output quickly enough to increase wind generation while maintaining load balance. The ramp-up constraints are caused by generators having to increase generation to meet the midday peak, and can similarly cause wind generation curtailments since conventional generators must be operated at their upper ramping limit in anticipation of the peak.

\footnotetext{
${ }^{9}$ The low wind utilization rates are due to exports from the West zone being binding both with and without RTP in every period of this particular day.
} 


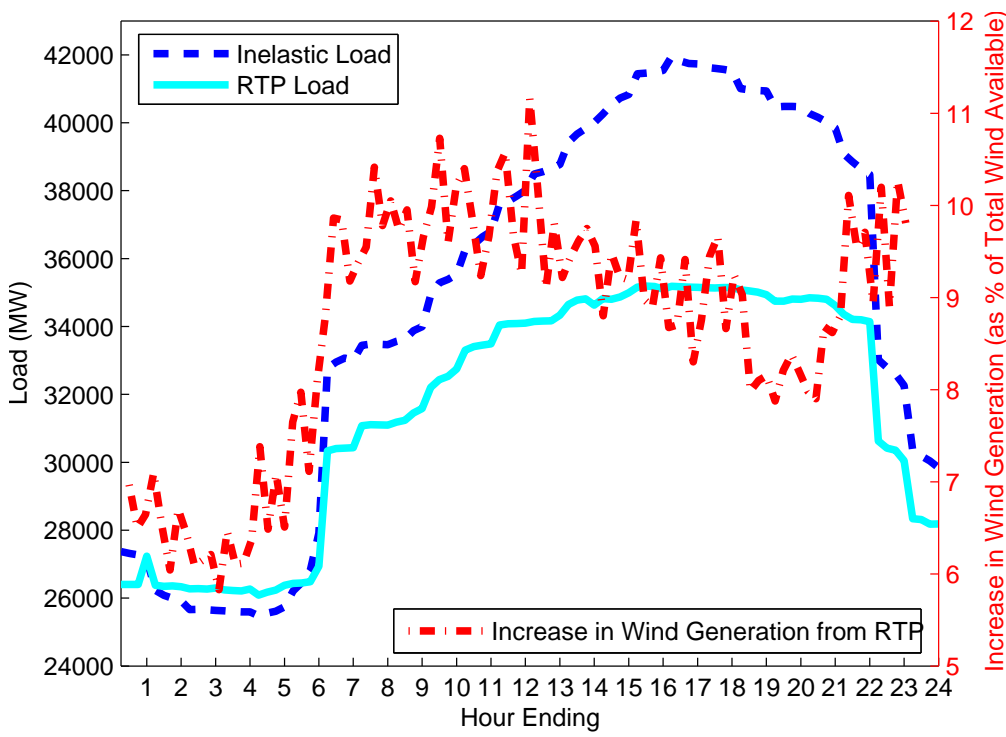

Figure 3: Diurnal Load Patterns With and Without Real-Time Pricing and Increase in Wind Generation.

Table 5: Number of Ramp-Down-Constrained Units in Each Hour

\begin{tabular}{l|ll} 
Hour & Fixed Load & RTP \\
\hline \hline 2 & 23 & 15 \\
3 & 12 & 0 \\
4 & 2 & 1 \\
20 & 1 & 0 \\
21 & 1 & 6 \\
22 & 2 & 0 \\
23 & 12 & 11 \\
24 & 50 & 22 \\
25 & 9 & 8 \\
\hline Total & 112 & 63
\end{tabular}

\subsection{Impact of RTP for C\&I Customers Only}

One impediment to the use of RTP in restructured markets has been the high cost of large-scale installation of interval meters that can record real-time electrical loads, and designing a system for communicating real-time prices to consumers 10 As such, some have advocated placing larger C\&I customers on RTP tariffs, since that would cover a large proportion of system load11 at a smaller implementation cost, and in some cases these efforts are already being undertaken 12 In California, for instance, all customers with a peak load above $200 \mathrm{~kW}$ are required to have

\footnotetext{
${ }^{10}$ Recent advances in metering and broadband over powerlines may reduce these costs to the point that large-scale installations would not be cost-prohibitive.

${ }^{11}$ For instance, C\&I customers accounted for nearly $63 \%$ of the load in Texas in 2005.

${ }^{12}$ Another rationale for keeping residential customers on fixed-rate tariffs is that RTP could expose customers to risk, volatility, and uncertainty in their retail electric rates, which may be too great for a small residential consumer to bear. Borenstein (2007) discusses the use of hedging instruments by a utility to insulate customers from most of this price risk, but subjecting them to marginal rates based on RTP to reap the efficiency gains.
} 
Table 6: Number of Ramp-Up-Constrained Units in Each Hour

\begin{tabular}{l|ll} 
Hour & Fixed Load & RTP \\
\hline \hline 4 & 3 & 0 \\
5 & 2 & 0 \\
6 & 8 & 1 \\
7 & 21 & 4 \\
8 & 32 & 12 \\
9 & 31 & 7 \\
10 & 8 & 9 \\
11 & 18 & 13 \\
12 & 2 & 17 \\
13 & 2 & 13 \\
14 & 0 & 11 \\
15 & 4 & 11 \\
16 & 3 & 14 \\
17 & 4 & 8 \\
18 & 1 & 6 \\
22 & 2 & 0 \\
23 & 6 & 0 \\
\hline Total & 147 & 126 \\
& &
\end{tabular}

15 -minute interval meters 13

We estimate how much of the gains from RTP can be achieved by applying it to these two customer segments only, by simulating the same set of unit commitment models assuming residential loads are fixed while C\&I demands are price-elastic. As discussed in section 2 we apportioned zonal loads in each time period to the three customer segments based on that segment's proportion of total electricity demand in Texas for 2005. Tables 7 and 8 summarize the results of our simulations with fixed residential loads, showing the percentage of the potential increases in wind generation which could be attained if all customers are placed on RTP that are achieved if only C\&I demands are made price-elastic (i.e. a value of 50 means half of the increase in wind generation from placing all customers on RTP are achieved if only C\&I customers are placed on RTP). These tables show that placing C\&I customers only on RTP reaps at least $60 \%$ of the gains from introducing variable tariffs for all customers, and may be a more practical starting point given the high costs of meter installation for smaller and residential customers.

\section{Conclusions}

We simulated and compared system operations with high wind penetration levels with and without RTP. Our results showed that even at low elasticity levels, RTP can increase both the percentage of load served by wind generation and the amount of potential wind generation actually utilized in real-time. Our results further show that RTP can increase wind utilization when transmission capacity is generally insufficient to export wind generation to load

\footnotetext{
${ }^{13}$ This cutoff is smaller for San Diego Gas and Electric (SDG\&E), since SDG\&E customers with a peak load above $100 \mathrm{~kW}$ are required to have interval meters.
} 
Table 7: Percentage of Increase in Average Wind Generation Utilization with RTP for all Customers Achieved with C\&I Customers Only on RTP

\begin{tabular}{ll|lll} 
& \multicolumn{4}{c}{ Wind Schedule Rating } \\
& & 0.1 & 0.5 & 1.0 \\
\cline { 2 - 5 } Elasticity & -0.10 & 64.03 & 64.45 & 64.84 \\
& -0.20 & 64.61 & 64.68 & 64.68 \\
& -0.30 & 65.48 & 65.67 & 65.57
\end{tabular}

Table 8: Percentage of Increase in Average Load Served by Wind Generation with RTP for all Customers Achieved with C\&I Customers Only on RTP

\begin{tabular}{ll|lll} 
& \multicolumn{4}{c}{ Wind Schedule Rating } \\
& & 0.1 & 0.5 & 1.0 \\
\cline { 2 - 5 } Elasticity & -0.10 & 80.65 & 82.35 & 83.78 \\
& -0.20 & 82.76 & 83.87 & 83.33 \\
& -0.30 & 85.37 & 84.88 & 84.78
\end{tabular}

pockets by using locational price differences to reduce congestion and increase wind utilization on the constrained side of a transmission link. This added impact of RTP may reduce the need for costly transmission investments. We also demonstrated that introducing RTP for industrial and commercial customers only can yield at least $64 \%$ of the potential gains from placing all three customer segments on RTP, which is potentially a more feasible alternative given the implementation costs and concerns over bill volatility with placing residential customers onto RTP tariffs.

Although these simulations are useful in determining the gains from RTP, they don't take into account all aspects of electricity markets or, in some cases, make simplifying assumptions. While it would be difficult to incorporate these characteristics into the modeling framework, it seems that with respect to these issues the analysis conducted here is potentially understating the gains from RTP.

Our simulations have ignored market power issues, and instead assumed the system will be perfectly competitive and generators committed and dispatched based on actual costs and with truthfully revealed constraint parameters. A number of studies, including Johnson, Oren, and Svoboda (1997) and Oren and Ross (2005), have demonstrated potential incentive issues with generators being able to profitably misstate their cost and constraint parameters when offering generation into a centrally-committed market. If one expects the exercise of market power to increase real-time prices, perhaps disproportionately in peak periods when supply is scarce, then our simulations may underestimate the extent to which RTP could flatten peaks and affect the load pattern. If the effect of market power is to increase real-time prices in all hours, however, then the net effect may be to shift loads down in all hours as opposed to having a pronounced effect on peaks or the load pattern.

Another simplifying assumption we made was that electricity demand under a RTP tariff would only exhibit own-price elasticities. Simulating a complete matrix of cross-price elasticities would increase model complexity substantially, as a 96-dimensional demand function (each dimension representing the price and load in each 15minute interval) would have to be approximated as a step function. As Borenstein (2005) discusses, this assumption is likely dampening the simulated effects of RTP. If demands are generally substitutes between hours then inclusion of cross-price elasticities would further smooth-out the load pattern by increasing off-peak and decreasing on-peak demands. This would, in turn, reduce the extent to which ramping and other operating constraints on conventional 
units impinge on the usage of wind generation.

\section{References}

Borenstein, S. (2002): "The Trouble With Electricity Markets: Understanding California's Restructuring Disaster," The Journal of Economic Perspectives, 16, 191-211.

(2005): "The Long-Run Efficiency of Real-Time Electricity Pricing," The Energy Journal, 26, 93-116.

(2007): "Customer Risk from Real-Time Retail Electricity Pricing: Bill Volatility and Hedgability," The Energy Journal, 28, 111-130.

Borenstein, S., and S. P. Holland (2005): "On the Efficiency of Competitive Electricity Markets With TimeInvariant Retail Prices," The RAND Journal of Economics, 36, 469-493.

Boresnstein, S., J. B. Bushnell, and C. R. Knittel (1997): "A Cournot-Nash Equilibrium Analysis of the New Jersey Electricity Market," Appendix A of Review of General Public Utilities Restructuring Petition, Final Report, New Jersey Board of Public Utilities, Docket Number EA97060396.

Denholm, P., and R. M. Margolis (2007): "Evaluating the limit of solar photovoltaics (PV) in traditional electric power systems," Energy Policy, 35, 2852-2861.

Hobbs, B. F., M. H. Rothkopf, R. P. O’Neill, and H.-P. Chao (eds.) (2001): The Next Generation of Electric Power Unit Commitment Models. Kluwer, Norwell, Massachusetts.

Johnson, R. B., S. S. Oren, and A. J. Svoboda (1997): "Equity and Efficiency of Unit Commitment in Competitive Electricity Markets," Utilities Policy, 6, 9-19.

Muckstadt, J. A., and S. A. Koenig (1977): "An Application of Lagrangian Relaxation to Scheduling in PowerGeneration Systems," Operations Research, 25, 387-403.

Oren, S. S., And A. M. Ross (2005): "Can we prevent the gaming of ramp constraints?," Decision Support Systems, 40, 461-471.

\section{A Model Formulation}

The formulation of the unit commitment model used in our simulations is presented. While the notation used here does not assume a specific time-step in making commitment and dispatch decisions, the relevant model parameters (e.g. ramp rates, minimum up- and down-times) are appropriately scaled for the day-ahead unit commitment with an hourly timestep and the real-time dispatch with 15-minute intervals. The AS constraints are only enforced for the day-ahead commitment models, not for the real-time dispatch models. Moreover, the binary commitment variables of all conventional generators, except those that provide non-spinning reserves while offline, are fixed in each realtime dispatch model based on the day-ahead unit commitment solution. Finally, the unit commitments with price inelastic demand were formulated with a fixed load, which could be equivalently represented within this formulation as a single-step demand function with an infinite price. We first define the following notation: 
Problem Parameters

- $T$ : number of periods

- $I$ : conventional generator index set

- $W$ : wind generator index set

- $Z$ : transmission zone set

- $L$ : transmission line set

- $C_{i}(q)$ : generator $i \in I$ 's non-decreasing stepped variable generating cost function

- $N_{i}$ : generator $i \in I$ 's noload cost

- $S U_{i}$ : generator $i \in I$ 's startup cost

- $K_{i}^{-}, K_{i}^{+}$: generator $i \in I$ 's minimum and maximum operating points, respectively

- $R_{i}^{-}, R_{i}^{+}$: generator $i \in I$ 's rampdown and rampup limits, respectively

- $\overline{S P}_{i}, \overline{N S}_{i}$ : generator $i \in I$ 's spinning and non-spinning reserve capacities, respectively

- $\tau_{i}^{-}, \tau_{i}^{+}$: generator $i \in I$ 's minimum down- and up-time, respectively

- $\omega_{w, t}$ : wind generation available from wind generator $w \in W$ in period $t \in T$

- $p_{z, t}(l)$ : non-increasing stepped inverse demand function of energy in zone $z \in Z$ in period $t \in T$

- $\eta^{s}, \eta^{n}$ : spinning and non-spinning reserve requirements (as a fraction of load), respectively

- $\rho$ : rating of day-ahead wind schedules in AS constraint

- $P T D F_{z, \lambda}$ : power transfer distribution factors between transmission zone $z \in Z$ and CSC $\lambda \in L$

- $T T C_{\lambda, t}$ : total transfer capacity on CSC $\lambda \in L$ in period $t \in T$

Decision Variables

- $q_{i, t}$ : generation provided by generator $i \in I$ in period $t \in T$

- $s p_{i, t}, n s_{i, t}$ : spinning and non-spinning reserves provided by generator $i \in I$ in period $t \in T$, respectively

- $u_{i, t}, s_{i, t}, h_{i, t}$ : binary variables indicating if unit $i \in I$ is up, started-up, and shutdown in period $t \in T$, respectively

- $g_{w, t}$ : wind generation provided by wind generator $w \in W$ in period $t \in T$

- $l_{z, t}$ : load served in transmission zone $z \in Z$ in period $t \in T$

- $\epsilon_{z, t}$ : net exports from transmission zone $z \in Z$ in period $t \in T$ 
The problem is formulated as maximizing social surplus:

$$
\max \sum_{z, t} \int_{0}^{l_{z, t}} p_{z, t}(x) d x-\left(\sum_{i, t} C_{i}\left(q_{i, t}\right)+N_{i} u_{i, t}+S U_{i} s_{i, t}\right) ;
$$

subject to the following constraints:

- zonal load-balance $(\forall z \in Z, t \in T)$ :

$$
l_{z, t}=\sum_{i \in I(z)} q_{i, t}+\sum_{w \in W(z)} g_{w, t}-\epsilon_{z, t},
$$

where $I(z)$ and $W(z)$ are conventional and wind generators located in zone $z \in Z$;

- no net exports $(\forall t \in T)$ :

$$
\sum_{z \in Z} e_{z, t}=0
$$

- total and spinning reserve requirements $(\forall t \in T)$ :

$$
\begin{gathered}
\rho \sum_{w \in W} g_{w, t}+\sum_{i \in I}\left(q_{i, t}+s p_{i, t}+n s_{i, t}\right) \geq\left(1+\eta^{n}\right) \sum_{z \in Z} l_{z, t} \\
\rho \sum_{w \in W} g_{w, t}+\sum_{i \in I}\left(q_{i, t}+s p_{i, t}\right) \geq\left(1+\eta^{s}\right) \sum_{z \in Z} l_{z, t}
\end{gathered}
$$

- conventional generator minimum and maximum generation bounds $(\forall i \in I, t \in T)$ :

$$
\begin{gathered}
K_{i}^{-} u_{i, t} \leq q_{i, t} \\
q_{i, t}+s p_{i, t} \leq K_{i}^{+} u_{i, t} \\
q_{i, t}+s p_{i, t}+n s_{i, t} \leq K_{i}^{+} ;
\end{gathered}
$$

- conventional generator AS bounds $(\forall i \in I, t \in T)$ :

$$
\begin{gathered}
0 \leq s p_{i, t} \leq \overline{S P}_{i} u_{i, t} \\
0 \leq n s_{i, t} \leq \overline{N S}_{i}
\end{gathered}
$$

- conventional generator ramping limits $(\forall i \in I, t \in T)$ :

$$
\begin{gathered}
R_{i}^{-} \leq q_{i, t}-q_{i, t-1} \\
q_{i, t}-q_{i, t-1}+s p_{i, t}+n s_{i, t} \leq R_{i}^{+}
\end{gathered}
$$

- conventional generator minimum up- and down-times $(\forall i \in I, t \in T)$ :

$$
\begin{gathered}
\sum_{y=t-\tau_{i}^{+}}^{t} s_{i, y} \leq u_{i, t} \\
\sum_{y=t-\tau_{i}^{-}}^{t} h_{i, y} \leq 1-u_{i, t} ;
\end{gathered}
$$


- conventional generator startup and shutdown state transitions $(\forall i \in I, t \in T)$ :

$$
\begin{gathered}
s_{i, t} \geq u_{i, t}-u_{i, t-1} \\
h_{i, t} \geq u_{i, t-1}-u_{i, t} ;
\end{gathered}
$$

- CSC flow limits $(\forall \lambda \in L, t \in T)$ :

$$
-T T C_{\lambda, t} \leq \sum_{z \in Z} P T D F_{z, \lambda} e_{z, t} \leq T T C_{\lambda, t}
$$

- wind generation bounds $(\forall w \in W, t \in T)$ :

$$
0 \leq g_{w, t} \leq \omega_{w, t}
$$

- non-negativity $(\forall z \in Z, t \in T)$ :

$$
l_{z, t} \geq 0 ; \text { and }
$$

- integrality of variables $(\forall i \in I, t \in T)$ :

$$
u_{i, t}, s_{i, t}, h_{i, t} \in\{0,1\} \text {. }
$$

